\title{
CONTEXTO SOCIAL Y FAMILIAR DESENCADENANTE DEL EMBARAZO EN ADOLESCENTES
}

\section{CONTEXTO SOCIAL E FAMILIAR DESENCADEADOR DA GRAVIDEZ EM ADOLESCENTES}

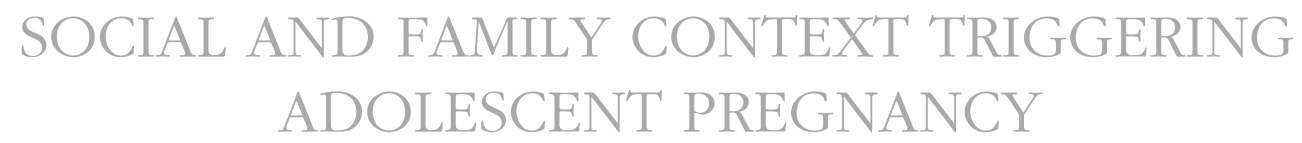

\begin{abstract}
Adriana Patricia López Benavides ${ }^{1}$
\end{abstract} Farlhyn Paola Bermúdez Moreno ${ }^{2}$

Como citar este artigo: Benavides APL, Moreno FPB. Contexto social y familiar desencadenante del embarazo en adolescentes. Rev baiana enfermagem. 2018 32:e24664.

Objetivo: comprender las características del contexto social y familiar desencadenantes del embarazo en adolescentes. Método: estudio de enfoque cualitativo, mediante entrevista a profundidad a 15 adolescentes entre 14 y 18 años en estado de gestación o con niños menores de 1 año, en el periodo de Mayo a Julio de 2017. La selección y ubicación de las participantes fue de tipo intencional en forma de bola de nieve. Resultados: se establecieron 4 categorías: relación con el entorno social, experiencia del embarazo, relación familiar y relación de pareja. Se evidenció que las adolescentes estan en una relación familiar disfuncional, la relación de pareja es inestable e inconstante, su entorno social es agresivo y descalificativo por su embarazo, aun así, manifestaron haber vivido el embarazo como una experiencia única y maravillosa. Conclusión: el contexto social y familiar está directamente relacionado con el embarazo en adolescentes, que es un fenómeno multifactorial complejo.

Descriptores: Adolescente. Embarazo en adolescencia. Relaciones familiares. Condiciones sociales. Acontecimientos que cambian la vida.

Objetivo: compreender as características principais do contexto social e familiar da gravidez em adolescentes. Método: estudo de enfoque qualitativo, mediante entrevista em profundidade com 15 adolescentes entre 14 e 18 anos gestantes ou com filhos menores de 1 ano, no período de maio a julbo de 2017. A seleção e localização das participantes foi intencional, sob a forma de bola de neve. Resultados: foram estabelecidas 4 categorias: relação com o meio social, a experiência da gravidez, relacionamento familiar e relacionamento com parceiro. Demonstrou-se que as adolescentes estão em uma relação familiar disfuncional, a relação com o parceiro é instável e inconstante, o ambiente social é agressivo e desqualificativo por causa da gravidez, mesmo assim, as participantes declararam ter vivido a gravidez como uma experiência original e maravilhosa. Conclusão: o contexto social e familiar é relacionado diretamente à gravidez na adolescência, aquela é um fenômeno multifatorial complexo.

Descritores: Adolescente. Gravidez na adolescência. Relações familiares. Condições sociais. Acontecimentos que mudam a vida.

Objective: to understand the characteristics of the social and family context triggering adolescent pregnancy. Method: a study with a qualitative approach, by means of in-depth interviews with 15 teenagers between the ages of 14 and 18 years, either pregnant or with children under the age of one, in the period of May to July 2017. The selection and location of the participants was of the purposive and snowball sampling type. Results: four categories were established:

Enfermera. Especialista enEpidemiología. Docente deEnfermería dela Fundación Universitaria delÁreaAndina. Pereira-Risaralda, Colombia. alopez3@areandina.edu.co Enfermera. Especialista en Auditoria y Calidad en Salud. Docente de Enfermería de la Fundación Universitaria del Área Andina. Pereira-Risaralda, Colombia. 
relationship with the social environment, experience of pregnancy, relationship with family and with the partner. It was evidenced that the adolescents are in dysfunctional family relations, the relationship with the partner is unstable and unsteady, social environment is aggressive and degrading due to the pregnancy, nevertheless they expressed the experience of pregnancy as unique and marvelous. Conclusion: the social and family context is directly related to adolescent pregnancy, a complex and multifactorial phenomenon.

Descriptors: Adolescent. Adolescent pregnancy. Family relations. Social conditions. Life changing events.

\section{Introducción}

La adolescencia es una etapa evolutiva del desarrollo humano que ocurre entre la infancia y la edad adulta, aproximadamente entre los 10 y los 19 años, en la cual acontecen un sinnúmero de cambios psicológicos, ambientales, sociales y biológicos, como lo son el alcance de la madurez sexual y reproductiva ${ }^{(1-2)}$. En este periodo se adquiere un nuevo estado de independencia en el cual se asumen nuevas responsabilidades y el adolescente inicia el arduo camino de reconocer su identidad, lo cual lo obliga a poner en práctica los valores aprendidos durante la infancia y a desarrollar nuevas habilidades y conductas que lo lleven a convertirse en un adulto responsable $e^{(3)}$.

La vivencia de la sexualidad como un proceso de asimilación de los cambios y procesos que sufre el cuerpo humano durante esta etapa no es tarea sencilla, más aún tratándose de los adolescentes de la actualidad, quienes aún no han tomado conciencia de la importancia que tiene adquirir una sexualidad responsable. Esto ha conducido a que en la actualidad el embarazo en la etapa de la adolescencia sea un problema de índole social, puesto que el embarazo es un acontecimiento que requiere de un esfuerzo adaptativo biológico y mental ${ }^{(4)}$, es decir de una madurez física y psicológica que conduzca a la vivencia plena de esta etapa, y las adolescentes por lo general no son poseedoras de dicha madurez ${ }^{(5-6)}$. Es por ello, que cuando las adolescentes interrumpen su vida cotidiana, con hechos tan relevantes como un embarazo, ven transformado su estilo de vida actual y futuro a largo plazo, lo cual en muchos casos genera grandes traumas en la vida tanto de la madre como del hijo. Sin embargo, en el caso de algunas adolescentes, la maternidad a edad temprana constituye una etapa que puede ser vivida plenamente, bien sea porque han planeado ser madres o porque asumen este nuevo rol de vida como algo positivo que transforma directamente su estilo de vida ${ }^{(7)}$.

Según la Organización Mundial de la Salud (OMS) y la Organización Panamericana de la Salud (OPS), unos 16 millones de adolescentes entre 15 y 19 años y aproximadamente 1 millón de niñas menores de 15 años dan a luz anualmente, la mayoría en países de medianos y bajos ingresos. La población entre los 15 y 19 años es la más propensa a presentar complicaciones durante el embarazo y el parto, lo cual constituye la segunda causa de muerte a nivel mundial. Se estima que cada año aproximadamente 3 millones de adolescentes en esta edad se somete a abortos, los cuales dejan graves secuelas tanto a nivel físico como psicológico y en los casos más graves ocasionan la muerte de la adolescente ${ }^{(8)}$.

El embarazo en la etapa de la adolescencia, es también considerado un problema de salud pública, Colombia presenta indicadores similares a los de países de Centroamérica y tasas mayores de fecundidad que en la mayoría de países de Suramérica $^{(9)}$. Por su parte, el comportamiento del embarazo adolescente en la ciudad de Pereira, durante los últimos 4 años ha mostrado una disminución, pasó de 1216 casos reportados en el año 2014 a 960 casos reportados en el año 2016, sin embargo, cabe destacar que el medio de verificación es el certificado de nacidos vivos, por lo tanto se desconoce si realmente ha disminuido la cifra de embarazos en adolescentes o sólo ha disminuido el número de nacimientos en este grupo etario ${ }^{(10)}$. 
Debido a que el embarazo en la adolescencia presenta un mayor riesgo de mortalidad y morbilidad tanto para la madre como para el hijo, cada vez aumentan más los gastos en materia de salud, ya que los hijos de madres adolescentes tienen mayor probabilidad de nacer prematuros, con bajo peso, y las madres mayores riesgos de desarrollar pre eclampsia, ruptura prematura de membrana, infección urinaria, anemia, sufrimiento fetal agudo, aborto o necesidad de una cesárea al momento del parto ${ }^{(11-15)}$.

El embarazo adolescente es un problema de tipo social que entre otras consecuencias, aumenta las tasas de deserción escolar, porque las adolescentes deben dedicarse a las labores del hogar y en muchos casos deben incurrir en la vida laboral antes de tiempo para garantizar el sostenimiento propio y el del hijo, lo cual disminuye las probabilidades de tener un trabajo digno y bien remunerado; genera familias monoparentales y familias disfuncionales, problemas de pareja, problemas con los hijos y problemas con la familia de origen ${ }^{(16-19)}$.

La familia juega un papel fundamental cuando de educación sexual y reproductiva se trata, de la educación que en esta se dé dependerán en gran medida el tipo de decisiones que las adolescentes tomen al iniciar su vida sexual, lo cual puede llevar a una considerable reducción del embarazo, el aborto y la disminución de enfermedades de transmisión sexual. Otros problemas a los que una buena educación familiar puede hacerle frente son: el alcoholismo, el consumo de sustancias psicoactivas y la delincuencia ${ }^{(16-17)}$. La familia como primera responsable y como institucion social debe empezar por reconocer su papel como guía y tutor de las adolescentes que están pasando por la etapa más compleja de sus vidas, una etapa en la cual no basta comprender, sino descubrir y dicho descubrimiento pasa por un periodo experimental.
Es necesario comprender las experiencias de las madres adolescentes desde el quehacer de la enfermería, ya que nos permite conocer e indagar el punto de vista de los actores sociales ${ }^{(20)}$, lo cual impacta de forma directa y positiva los servicios de salud, puesto que al comprender las experiencias de los otros se puede brindar una atención más personalizada y tendiente a mejorar la calidad de la vida de las pacientes. El fenómeno del embarazo es por lo tanto un fenómeno multifactorial en el cual influyen factores como: la familia y los patrones de crianza, la sociedad, la escuela y el uso del tiempo libre. El presente artículo tiene por objetivo comprender las características del contexto social y familiar desencadenantes del embazado en adolescentes.

\section{Método}

Se realizó un estudio de enfoque cualitativo con el fin de realizar un análisis descriptivo en base a las experiencias compartidas, las vivencias y los significados que les atribuyen las adolescentes al embarazo, para determinar como el contexto social y familiar pueden ser desencadenantes del embarazo en adolescentes de la ciudad de Pereira, Colombia.

La población estudiada estuvo conformada por adolescentes entre 14 a 18 años pertenecientes al estrato socioeconómico 1 y 2 en estado de embarazo o con hijos menores de 1 año, a las cuales se les realizaron entrevistas en el periodo comprendido entre mayo y julio de 2017. La selección y ubicación de las participantes fue intencional, usando otras estrategias como la bola de nieve, donde el enlace inicial fueron las adolescentes gestantes que asistieron a la estrategia de Cero a Siempre en la ciudad de Pereira ${ }^{a}$.

Se diseñó una entrevista a profundidad semiestructurada con una duración aproximada de 45 minutos, que se aplicó de manera individual

\footnotetext{
La Estrategia De Cero a Siempre fue aprobada como Ley de la República y sancionada por el Presidente de la República, el 2 de agosto de 2016 . En consecuencia, la atención integral a la primera infancia deberá ser implementada en todo el País, logrando avanzar en condiciones reales en favor del desarrollo integral de niñas y niños. Los derechos de las niñas y los niños en primera infancia son impostergables; la familia, la sociedad y el estado están en la obligación de garantizar la protección, la salud, la nutrición y la educación inicial desde el momento de la gestación hasta los cinco años. La Estrategia De Cero a Siempre coordinará todas las instituciones, tanto públicas como privadas, para lograr garantizar, en el largo plazo, una atención integral a 2'875.000 niños y niñas del Sisbén I, 2 y 3. Trabajando bajo una perspectiva de universalización de la Atención Integral, De Cero a Siempre priorizará la población en Pobreza Extrema. En la actualidad sólo el $24 \%$ los niños y niñas menores de cinco años recibe atención integral, y De Cero a Siempre buscará hacer efectivos los derechos a la atención integral de I'500.000 niños y niñas en situación de vulnerabilidad, enfrentándose así a un gran reto Nacional que exige una coordinación interinstitucional para alcanzar una cobertura del I00\% de la población entre cero y cinco años de edad. Available from: http://www.deceroasiempre.gov.co/QuienesSomos/Paginas/QuienesSomos.aspx
} 
a las adolescentes gestantes y a las madres adolescentes de niños menores de 1 año de las comunas de Villa Santana, Altagracia y el sector de Cuba en los barrios El Dorado y Salamanca de los estratos socioeconómicos 1 y 2 , para lo cual los padres de las adolescentes participantes firmaron un consentimiento informado y las adolescentes dieron su asentimiento para participar de la investigación. Las entrevistas fueron realizadas en las casas de cada participante, para lo cual se requirió desplazarse a cada ubicación indicada por las adolescentes.

Se grabaron un total de 15 entrevistas conforme al número de participantes, para disponer de un registro fidedigno del material recolectado, después se procedió con la transcripción de las entrevistas de forma textual, cuidándose de registrar todo lo que se dijo en el audio. El criterio para la interrupción de la entrevista se determinó al abarcar todos los interrogantes previamente establecidos que fueron necesarios para conocer a profundidad los criterios a analizar. Las participantes de la presente investigación pueden ser identificadas en el texto con la letra E de Entrevista y el número asignado a cada entrevista.

El análisis de la información de la entrevista se basó en los testimonios, debido a que éstos permiten extraer significados útiles al objetivo de la investigación; las categorías de análisis se establecieron previamente, a partir de la revisión bibliográfica de los elementos que podrían describir mejor el contexto familiar y social, de lo cual surgieron cuatro categorías: relación familiar, relación de pareja, relación con el entorno social y experiencia del embarazo. Para analizar el contexto familiar se establecieron dos categorías: relación familiar y relación de pareja y para el contexto social dos categorías: relación con el entorno social y experiencia del embarazo.

Dado que el presente artículo obedece a un proyecto institucional, los aspectos éticos se consideran en el Comité de Investigación de la Universidad Andina, el cual es garante del cumplimiento de los aspectos éticos del trabajo.

\section{Resultados y Discusión}

El embarazo en la adolescencia es un fenómeno de causa multifactorial que ocurre en todos los estratos socioeconómicos, sin embargo es en los niveles socioeconómicos más bajos donde hay mayor riesgo de embarazarse a edad temprana, además son más frecuentes los embarazos no deseados y la falta de cuidados prenatales, lo cual aumenta la posibilidad de detectar de manera temprana los factores de riesgo, lo cual resulta en una mayor morbimortalidad materna y perinatal ${ }^{(6-10,12)}$. Además en la mayoría de los casos constituye un problema de tipo social, que hace que la madre interrumpa su vida escolar para dedicarse a los cuidados de su hijo o a trabajar para el sostenimiento de ambos ${ }^{(7-21)}$.

Las categorías relación con el entorno social y experiencia del embarazo representan el eje para comprender las características del contexto social y las categorías relación familiar y relación de pareja permiten comprender el contexto familiar y como desencadenantes del embazado en adolescentes en la ciudad de Pereira.

\section{Relación con el Entorno Social}

El acelerado desarrollo sexual y reproductivo de las adolescentes de la actualidad, obliga a que se le atribuya una mayor importancia a la educación sexual tanto en el ámbito familiar como en el escolar y el social, explicando y aclarando sus dudas sobre sexualidad, métodos de planificación familiar, enfermedades de transmisión sexual y embarazo, enunciando todas sus implicaciones tanto para la madre como para el hijo ${ }^{(11)}$.

La maternidad en la adolescencia implica asumir a temprana edad un nuevo rol social, el de madre, este papel exige que las adolescentes adquieran un nivel de responsabilidad nunca imaginado con respecto al hijo que se está gestando o al bebé por el cual deben responder.

Cómo le digo yo, no es un trabajo fácil porque yo soy una niña no soy ya una madre adulta entonces es como dificil, pero igual uno comete los errores y tiene que afrontarlos, y pues mi mamá y mi papá me han apoyado mucho, y por ellos fue que no tomé una mala decisión. (E2). 
Las investigaciones señalan que existe un fuerte vínculo entre los casos de embarazo y los de abandono escolar, ya que el embarazo a edad temprana incrementa los problemas y dificultades en el manejo de las situaciones cotidianas y eventuales, como las relacionadas con el ámbito escolar y el futuro laboral; condiciones que agudizan problemas como la inequidad de género ${ }^{(16,22-24)}$. Esto se ve reflejado en el hecho de que las adolescentes embarazadas se ven obligadas a abandonar sus estudios; sin embargo, 10 de las adolescentes encuestadas aún se encuentra estudiando, aunque sólo 2 de ellas en la jornada regular, las otras 8 adolescentes estudian en la nocturna o los fines de semana; cabe resaltar que muchas de ellas ya estudiaban en estas jornadas antes de enterarse de su embarazo. Las otras 5 jóvenes actualmente se dedican a las labores del hogar y a la crianza de sus hijos; a su vez manifiestan falta de deseo por continuar sus estudios, ya que se encontraban fuera del ámbito escolar antes de quedar embarazadas y les produce vergüenza que las personas vean su estado; puesto que aún son incapaces de entender que la permanencia en la escuela favorece su desarrollo personal y les permite tener roles sociales distintos al que les otorga la maternidad $^{(17)}$.

No, yo antes de quedar en embarazo me sali del colegio, unos tres meses antes [...] No, yo digo que las amistades, yo era con mis amigas y mis amigas ya no me gustaba el estudio, entonces por eso fue, no fue el embarazo, antes, por ahi unos tres meses, me sali del colegio. (E2).

De acuerdo a otros trabajos, las adolescentes que abandonan sus estudios tempranamente $\mathrm{y}$ se dedican a las labores del hogar, tienen mayores riesgos de embarazarse tempranamente, con respecto a las que se ocupan en actividades académicas, ya que el nivel educativo es indispensable para la toma de decisiones y se observa con frecuencia como en la literatura se relaciona esta variable con el conocimiento y uso de métodos de planificación familiar y mayor responsabilidad frente al inicio de la vida sexual ${ }^{(25)}$. Las adolescentes sin diferenciación entre las que están estudiando y las que abandonaron sus estudios, manifiestan que tras su embarazo y maternidad vieron transformado su proyecto de vida.

Pues, yo pienso que no ha cambiado mucho, sólo que tengo un bebé y ya es más responsabilidad, yo pensaba bacer una carrera que no se podia tener bijos, quería ser azafata, pero yo pienso estudiar, meterme al SENA o a una universidad. (E6).

Tales resultados nos permiten afirmar que el contexto social en que las adolescentes viven influye de manera directa en su conducta sexual y reproductiva, en los roles que desempeñan, en los encuentros sexuales y en la toma de decisiones sexuales y reproductivas ${ }^{(22)}$, siendo menor el grado de discernimiento de las adolescentes que crecen en barrios de nivel socioeconómico más bajo, lo cual se ha reflejado en otros trabajos, donde se ha concluido que existe una importante relación entre la pobreza, la inequidad y la desigualdad, con el embarazo antes de los 15 $\operatorname{años}^{(24)}$, lo que tiende a incrementar la pobreza, la ausencia de oportunidades y la desnutrición infantil $^{(5)}$.

\section{Experiencia del Embarazo}

Los hijos deberían ser los miembros de la familia que llegan a reforzar los lazos fomentados por la unión de pareja: hombre y mujer, consolidando con ello la nueva relación: padre y madre, con el objeto de cimentar las bases de desarrollo social, sin embargo, en la actualidad y a causa entre otras cosas del fenómeno del embarazo adolescente ya no lo son.

Pues yo no sufrí tanto en el embarazo, yo siempre quise ser mamá joven, como a los 20 algo así, pero fue pues a los 15, el embarazo fue muy sufrido fue por el papá de la niña, porque él me dejó cuando yo tenía 2 meses. (E7).

El embarazo en la adolescencia tiene grandes implicaciones en la salud pública, convirtiéndose en un hecho preocupante desde el punto de vista médico como psicosocial, siendo un fenómeno de mayor frecuencia en las adolescentes de nivel socioeconómico más bajo, en familias disfuncionales, afectadas por el desempleo y la violencia ${ }^{(11,14)}$. Las investigaciones señalan la relación directa que existe entre los casos de embarazo adolescente y las dificultades de las mujeres pára acceder a la educación superior, 
ya que es en la etapa de la adolescencia donde se da inicio a la proyección de la vida futura, y dicha proyección se ve seriamente afectada cuando las adolescentes deben trabajar para hacerse cargo de un hijo ${ }^{(22-23)}$.

Entre las adolescentes 13 manifiestan tener cierto conocimiento de métodos de planificación, sin embargo, afirman no haberlo usado por razones como subida de peso o cambios hormonales, otras usaban preservativo, pero de manera ocasional pues creían que no iban a quedar embarazadas.

Ah, ab ¿el método? Ah, no, el condón, muchas veces se rompia [...] Sí, claro. Dicen que cuando uno toma muchas pastas ese dizque del día después uno queda estéril, y yo ya habia tomado varias porque habia pasado pues varios susticos, y pues no [risas]. (E7).

Es de notar la confusión que existe entre las adolescentes sobre los métodos de planificación, esto es bastante evidente en otras investigaciones donde es casi nulo el uso de anticonceptivos en las adolescentes menores de 15 años, bien sea por restricción familiar, por el poco acceso a consultas y asesoría en anticoncepción y salud sexual y reproductiva ${ }^{(24)}$.

Aunque la mayoría de ellas manifiesta que el embarazo fue accidental, asumen el cuidado y la crianza de su hijo como una responsabilidad propia $^{(20)}$ y afirman sentirse apoyadas por sus familias.

Pues mucha felicidad, al principio pues muy sorprendida porque no me lo esperaba, pero la verdad me be dado cuenta que un bebé es una bendición de Dios, ha unido mucho a mi familia, han estado conmigo mucho. (E1).

Es evidente la ambigüedad emocional que trae para las adolescentes estar embarazadas y asumir como su vida se ha transformado al convertirse en madres a edad temprana, esto se ha reflejado en otros trabajos donde las adolescentes indican que debieron esperar más tiempo para embarazarse, ya que un hijo requiere de muchas cosas que ellas no les pueden dar, entre otras cosas el factor económico, sin embargo asumen su responsabilidad como madres de manera consciente y no se arrepienten de serlo ${ }^{(24)}$.

Pues es algo muy lindo, pero también significa que le quita a uno muchas posibilidades, por ejemplo, cuando yo me di cuenta que estaba embarazada tenía como cuatro meses y estaba aún yendo al gimnasio, como no me dieron sintomas entonces yo iba, entonces dejar de lado una rutina que uno ya tení, a veces uno se le olvida, uno no puede correr. (E15)

Entre las adolescentes embarazadas se reporta normalidad en la gestación, sólo una de ellas manifestó haber sufrido de complicaciones durante su gestación, aunque las mismas fueron fácilmente controladas en los servicios de urgencias.

Esto difiere de lo establecido por algunas investigaciones, donde es común encontrar reportes de complicaciones en embarazos adolescentes que implican un alto riesgo para la madre y el bebé, como son anemia, hipertensión, abortos, amenazas de abortos y/o de parto pretérmino, infecciones de vías urinarias y vaginosis, recién nacidos con bajo peso, morbilidad y mortalidad neonatal. Apgar bajo, otras complicaciones neonatales, incluyendo un nuevo embarazo ${ }^{(10-12)}$. La comprensión de los riesgos de un embarazo en la etapa de la adolescencia es indispensable para reducir los índices de maternidad en adolescentes y sus complicaciones.

\section{Relación Familiar}

La familia debería ser lo más importante para los seres humanos, lamentablemente muchos padres y madres no lo consideran así y se escudan en sus múltiples ocupaciones para justificar el deterioro de la misma. Se encontró que, entre las adolescentes encuestadas, el tipo de familia que prevalece es la familia de padres separados, donde sólo 7 de las encuestadas manifiesta tener un hogar completo formado por padre y madre, de las cuales sólo 4 viven una relación familiar armónica.

Ah, con mi mamá, mi papá, mis sobrinos, y con, o sea ellos todos son un apoyo. (E2).

Como resultado de esto podríamos afirmar, que los hijos de padres divorciados tienen mayores probabilidades de presentar problemas de adaptación a la vida social; es evidente como la ausencia de uno de los padres puede constituir un factor de riesgo para el embarazo adolescente. Sin embargo, los efectos psicológicos 
negativos en la personalidad de los hijos no sólo se deben a la separación de los padres, también influyen factores como las malas relaciones al interior de la familia, las relaciones de pareja poco sanas y la violencia intrafamiliar, ya que los adolescentes requieren de una estabilidad afectiva y emocional que puede verse seriamente amenazada cuando crecen en el seno de familias disfuncionales.

Jun., pues muy dura porque mis papás se separaron y nosotros estábamos muy chiquitos, entonces pues casi toda mi infancia fue de aquí para allá y de allá para acá, un tiempo con mi papá, otro tiempo con mi mamá, entonces siempre da muy duro eso. (E4).

La etapa adolescente es una etapa de cambios periódicos, reflejados en los planos físico, psicológico y social del individuo, los cuales generan tensiones que alteran la inserción social. Esta etapa es considerada como un periodo transitivo entre la infancia y la adultez, por ello la disfunción familiar es un factor que predispone la aparición de trastornos emocionales y psicológicos, expresados en conductas dañinas como la farmacodependencia, la deserción escolar, la depresión, el suicidio, el inicio prematuro de la vida sexual, las enfermedades de transmisión sexual y el embarazo adolescente, entre otros ${ }^{(2,16-17)}$.

El sistema familiar provee al adolescente de distintos elementos culturales y normas sociales que permiten su integración a la sociedad, por lo tanto, podría afirmarse que las adolescentes que no tienen buenas relaciones familiares o que provienen de familias disfuncionales corren mayores riesgos de presentar un embarazo a edad temprana, ya que su educación en materia sexual y reproductiva en la mayoría de los casos es insuficiente. Las investigaciones resaltan la relación que existe entre las pautas de crianza y el inicio de la vida sexual y reproductiva a edad temprana; esto porque los padres de las adolescentes presentan inconsistencias en el cuidado de sus hijas, ya que al tratar de cuidarlas se van bien sea por uno de los dos extremos: las cuidan demasiado o no saben cuidarlas ${ }^{(19)}$ y esto conduce a una vivencia incorrecta de su libertad y su sexualidad.
La ausencia de diálogo y confianza con la familia en temas relacionados con la vida sexual y reproductiva y en los demás aspectos de su vida, se refleja en la poca interacción familiar de las adolescentes, las cuales a la hora de decidir que hacer con su embarazo y frente al abandono de la pareja, se ven obligadas a buscar apoyo en terceros, mientras los padres se muestran despreocupados por las actividades de las adolescentes $^{(18)}$.

Ah, porque yo tenía muchas amigas que me decian que
ah que abortara, y no, yo no fui capaz, yo me vine a don-
de mi mamá, yo le dije que no era capaz, ella me dijo
pues que es que eso no era, que yo no era dueña de la
vida de nadie como para matar a una criatura y que
ellos me iban a colaborar. (E2).

Sin embargo, las adolescentes afirman que pese a que los padres se disgustaron mucho frente a la noticia de su embarazo, terminaron por apoyarlas económica y emocionalmente, aunque dicho apoyo prevalece por parte de la madre, a quien ven como un apoyo esencial para compensar la falta de habilidades para llevar el embarazo adecuadamente, para la crianza y como apoyo económico ${ }^{(7)}$, puesto que las familias tienen un nivel de adaptabilidad frente a los hechos de estrés situacional buscando siempre mejorar su capacidad de adaptabilidad y progreso para enfrentar estas condiciones ${ }^{(2,18)}$.

No pues antes me daba mucho gusto, yo era la niña de
él, y abora también. Ya está muy feliz con la niña y pues
a mí tampoco me ha dejado de consentir, el me da pues
todo, y pues la niña es la adoración de él, y con mi mamá
también. Al principio a él no le importaba que me fuera,
por la rabia, pero ya después cuando me vine para acá
mejoró la relación. (E13).

\section{Relación de Pareja}

Tomar la decisión de vivir en pareja o de unirse en matrimonio en torno a la creación de una nueva familia no es tarea sencilla, es una decisión que debe partir de un análisis serio, donde se definan los pro y los contra, de realizarse bajo este argumento, se garantiza una convivencia sana, en la cual, aunque existan dificultades como es normal, pueden ser superadas con apoyo mutuo. Los resultados de este 
estudio muestran que el embarazo adolescente no obedece a un proceso de toma consciente de decisiones entre los dos miembros de una pareja, antes bien, es la consecuencia de la falta de conocimiento y la no utilización de métodos anticonceptivos o de su uso incorrecto.

Yo estuve con él como seis meses no más, y en el embarazo hasta los cuatro meses [...] Pues yo planifiqué con él con preservativo no más, y ya cuando no abi fue que quedé embarazada. (E6).

Sin embargo, los embarazos de estas adolescentes no son el resultado de actos sexuales esporádicos, antes bien suceden bajo relaciones de noviazgo previamente establecidas, en las cuales se dio inicio a la actividad sexual de manera $\operatorname{precoz}^{(7)}$.

Vamos a cumplir dos años [...] No, cuando nos fuimos a vivir juntos, él y yo queríamos tener un bebé [...] A los diez meses quedé en embarazo. (E8).

Pues yo quería terminar mi estudio, pero él quería [...] No pues los dos queríamos, pero el pensado mí era terminar primero mi estudio para después quedar en embarazo. (E9).

En la literatura se asocia la convivencia o estar casados con el embarazo, con frecuencia se relacionan los deseos de tener hijos en la adolescencia con las relaciones de pareja de unión libre o matrimonio ${ }^{(25)}$. Con respecto a la participación de la pareja, esta inicialmente asume una postura de miedo; posteriormente asume una de dos conductas, presencia o ausencia; la mitad de las participantes afirman tener algún apoyo del padre del bebé, aunque no convivan juntos y la otra parte de ellas afirman encontrarse solas al momento del embarazo, o no tener relación alguna con el padre de sus hijos.

Íbamos para un año, él venía acá, charlaba con mis hermanos, él tiene 19 años [...] Sí, él se fue hace como cuatro meses, él sabe que estoy embarazada [...] Él es un muchacho pues sano, a la vez no, casi no le gusta trabajar, y ya. Él trabajaba cuando yo lo conocí, en obra blanca, constructor. Me ayudaba me daba dinero, a lo último no. (E14).

El apoyo recibido por parte de la pareja es algo muy importante para las adolescentes, ya que consideran que la aceptación social de su embarazo depende en gran medida de la relación que tienen con el padre de su hijo ${ }^{(7)}$. Sin embargo, uno de los factores más significativos en las relaciones de pareja de las adolescentes que actualmente están asumiendo su maternidad sin apoyo de una figura paterna, es que dichas relaciones no duraron más de un año.

Limitaciones y fortalezas del estudio: durante la realización del estudio se pudo observar por parte de las adolescentes y de sus padres cierta reticencia para participar de la investigación, además algunas de las jóvenes abordadas para el estudio manifestaron vergüenza por su estado de gestación. El medio de verificación de embarazos adolescentes es el certificado de nacidos vivos, lo cual impide conocer a profundidad el fenómeno del embarazo en Pereira, Colombia.

\section{Conclusión}

El embarazo en adolescentes es un fenómeno multifactorial complejo, entre sus muchas causas resaltamos hogares o familias disfuncionales, falta de educación sexual, contextos sociales poco favorecedores y exploración de la sexualidad a edad temprana.

Existen elementos tanto del contexto social como del familiar que se reflejan en los testimonios de las adolescentes que pueden ser desencadenantes del embarazo en adolescentes. En el contexto social, existe una amplia relación entre el abandono escolar y los casos de embarazo a edad temprana, ya que el nivel educativo es indispensable a la hora de tomar decisiones y vivir responsablemente la sexualidad. Por otra parte, los roles que desempeñan las adolescentes que se embarazan a edades tempranas, tienden a aumentar las brechas entre la pobreza y la riqueza, ya que estas debido a la necesidad de sostenimiento de ellas y sus hijos se ven obligadas a aceptar trabajar mal remunerados.

En el contexto familiar, entre los factores desencadenantes del embarazo adolescente, se destacan las familias disfuncionales, compuestas en su mayoría por uno sólo de los padres. Otro factor que influye en el inicio de la vida sexual a edad temprana y los embarazos en la adolescencia es la falta de confianza y el poco diálogo con los padres. 
Conocimiento desde la fuente de las percepciones de las adolescentes sobre el embarazo a edad temprana y la influencia que ejerce el contexto familiar y social en el inicio de la vida sexual y reproductiva. Este estudio sirve como punto de partida para realizar un control preventivo del embarazo en adolescentes, basados en las vivencias y experiencias aquí registradas.

Se recomienda que se planteen nuevas investigaciones cualitativas acerca del embarazo en adolescentes, realizando comparaciones entre grupos focales de gestantes adolescentes y analizando los diferentes fenómenos sociales que inciden en el embarazo a edad temprana como lo son las características socioeconómicas y el nivel educativo de la población estudiada. Se recomienda que las autoridades gubernamentales a nivel local, municipal y departamental planteen estrategias para la identificación de poblaciones vulnerables y con mayor riesgo de embarazarse a edad temprana, y a partir de los resultados generen nuevas estrategias para contrarrestar el embarazo adolescente como los son las campañas de educación sexual.

\section{Colaborações:}

1. concepção, projeto, análise e interpretação dos dados: Adriana Patricia López Benavide e Farlhyn Paola Bermúdez Moreno;

2. redação do artigo e revisão crítica relevante do conteúdo intelectual: Adriana Patricia López Benavide e Farlhyn Paola Bermúdez Moreno;

3. aprovação final da versão a ser publicada: Adriana Patricia López Benavide e Farlhyn Paola Bermúdez Moreno.

\section{Agradecimientos}

A las adolescentes participantes del estudio por la acogida, la confianza y por permitirnos compartir con ellas, así fuese de manera limitada, algo de su vida y experiencia de ser adolescentes y estar embarazadas.

Queremos agradecer al Dr. Andrés Alonso Agudelo Suárez, por el Acompañamiento y
Algunos Aportes durante la Elaboración del trabajo de Investigación.

\section{Referencias}

1. Diverio IS. La adolescencia y su interrelación con el entorno. Madrid (ES): Instituto de la Juventud; 2007 [cited 2017 Oct 25]. Available from: http://www.injuve.es/sites/default/files/LA\%20 ADOLESCENCIA $\% 20 y \% 20 \% 20$ entorno_completo. pdf

2. Calderón IC, Del Río CI, Rodríguez O, Guisandes AA. Disfunción familiar en gestantes adolescentes de un área de salud. Medisan [Internet]. 2014 Abr 17 [cited 2017 Oct 25];18(11):1544-9. Available from: http://scielo.sld.cu/scielo.php?script=sci_ arttext\&pid=S1029-30192014001100009

3. Unicef. Adolescencia una etapa fundamental. New York: Unicef; 2002 [cited 2017 Oct 25]. Available from: https://www.unicef.org/ecuador/ pub_adolescence_sp.pdf

4. López N. ¿Cómo cambia el cerebro un aborto inducido? Cuad Bioet [Internet]. 2012 [cited 2017 Nov 5];23(2):565-84. Available from: http:// aebioetica.org/revistas/2012/23/78/565.pdf

5. Gaete V. Desarrollo psicosocial del adolescente. Rev Chil Pediatr [Internet]. 2015 Sep 2 [cited 2017 Nov 11];86(6):436-43. Available from: https:// ac.els-cdn.com/S0370410615001412/1-s2.0S0370410615001412-main.pdf?_tid=70f11b80-c7e711e7-a051-00000aacb35f\&acdnat=1510518430_8aa 56d03f381f36a922a26eb7f7ea13d

6. Eslava DG, Rodríguez SV. "Estamos muy jóvenes para tener hijos": vivencias de la planificación familiar de un grupo de adolescentes en un municipio de Risaralda. Investig Enferm. Imagen Desarr [Internet]. 2008 jul/dic [cited 2017 Nov 11];10(2):115-32. Available from: http://revistas. javeriana.edu.co/index.php/imagenydesarrollo/ article/viewFile/1605/1028

7. Alvarez C, Pator G, Linares M, Serrano J, Rodríguez L. Motivaciones para el embarazo adolescente. Gac Sanit [Internet]. 2012 dic [cited 2017 Nov 6];26(6):487-503. Available from: http://scielo.isciii.es/scielo.php?script=sci_ arttext\&pid=S0213-91112012000600002

8. Cajina JC. El embarazo en adolescentes. Boletín informativo. Ginebra: Organización Panamericana de la Salud; Organización Mundial de la Salud; 2015 [cited 2017 Nov 10]. Available from: http:// 
www.paho.org/nic/index.php?option $=\mathrm{com}_{-}$ docman\&view=download\&category_slug=datos$\mathrm{y}$-estadisticas\&alias $=714$-boletin-informativoembarazo-en-adolescentes\&Itemid $=235$

9. Gallego GA. Análisis de la línea base del Observatorio de Embarazo Adolescente en Colombia. Colombia: Ministerio de Salud y Protección Social; 2015 [cited 2017 Nov 20]. Available from: https://www.minsalud.gov.co/ sites/rid/Lists/BibliotecaDigital/RIDE/VS/ED/ GCFI/ONIEA_003_Informe_Linea\%20Base $\% 20$ ONIEA_02_08_2013.pdf

10. Alcaldía de Pereira. Pereira cómo vamos. Informe de calidad de vida 2016. Pereira: Alcaldía de Pereira; 2016 [cited 2016 Nov 21]. Available from: http://www.pereiracomovamos.org/es/dominios/ pcv.pagegear.co/upload/69/2017/informe_de_ calidad_de_vida_2016_v.04.pdf

11. Zamora AN, Panduro JG, Pérez JJ, Quezada NA, Gónzalez J, Fajardo S. Embarazo en adolescentes y sus complicaciones materno perinatales. Rev Med [Internet]. 2013 ago 1 [cited 2017 Oct 27];4(4):233-8. Available from: http://www.medigraphic.com/ pdfs/revmed/md-2013/md134e.pdf

12. Sáez V. Morbilidad de la madre adolescente. Rev Cubana Obstetricia Ginecología [Internet]. 2005 ago [cited 2017 Oct 30];31(2):1-7. Available from: http://scielo.sld.cu/scielo.php?script $=$ sci_ arttext\&pid=S0138-600X2005000200003

13. Mendoza LA, Arias M, Mendoza LI. Hijo de madre adolescente: riesgos, morbilidad y mortalidad neonatal. Rev Chil Obstetricia Ginecología [Internet]. 2012 [cited 2017 Oct 30];77(5):375-82. Available from: http://www.scielo.cl/scielo.php?script=sci_ arttext\&pid $=$ S0717-75262012000500008

14. Izaguirre $=$ González A, Aguilar-Reys V, RamírezUzcoa A, Valladares-Rivera G, Rivera-Mejía W, Valladares-Rivera E, et al. Incremento de riesgo obstétrico en embarazo adolescente. Estudio de casos y controles. Arch Med. 2016 oct 29 [cited 2017 Oct 30];12(4):1-6. DOI: 10.3823/1326

15. Donoso E, Carvajal JA, Vera C, Poblete JA. La edad de la mujer como factor de riesgo de mortalidad materna, fetal, neonatal e infantil. Rev Med Chile [Internet]. 2014 enero 16 [cited 2017 Oct 30];142(2):168-74. Available from: http://www. scielo.cl/pdf/rmc/v142n2/art04.pdf

16. Trillo V, Loreto N, Figueroa C. Embarazo en adolescentes: problema de salud pública o de intervención social. Culcyt [Internet]. 2013 enero [cited 2017 Nov 1];10(49):51-60. Available from: http://erevistas.uacj.mx/ojs/index.php/culcyt/ article/view/140/138

17. Villalobos A, Campero L, Suárez L, Atienzo EE, Estrada F, De la Vara E. Embarazo adolescente y regazo educativo: análisis de una encuesta nacional en México. Salud Pública Mex [Internet]. 2015 marzo [cited 2017 Nov 5];57(2):135-43. Available from: http://www.scielosp.org/pdf/spm/ v57n2/v57n2a8.pdf

18. García GE. Embarazo adolescente y pobreza, una relación compleja. Itzap Rev Cien Soc Hum [Internet]. 2014 jul [cited 2017 Nov 3];77(35):13-53. Available from: http://www.redalyc.org/articulo. oa?id=39348246002

19. Calderón J, Alzamora L. Influencia de las relaciones familiaressobreelabortoprovocadoenadolescentes. Rev Perú Med Exp Salud Pública [Internet]. 2006 [cited 2017 Nov 6];23(4):247-52. Available from: http://www.scielo.org.pe/scielo.php?script=sci_ arttext\&pid=S1726-46342006000400003

20. Gonçalves M. Los adolescentes como agentes de cambio social: algunas reflexiones para los psicólogos sociales comunitarios. Psykhe [Internet]. 2004 nov [cited 2017 Nov 11];13 (2):131-42. Available from: http://www.scielo.cl/scielo.php?script=sci_ arttext\&pid=S0718-22282004000200010

21. Pacheco CI. Agencia social, sexualidad y embarazo en menores de 15 años. Rev Gerenc Polit Salud [Internet]. 2015 dic [cited 2017 Nov 7];14(29):62-82. Available from: http:// www . scielo.org.co/scielo.php?script $=$ sci $_{-}$ arttext\&pid=\$1657-70272015000200005

22. Chávez AM, Landa A, Menkes C, Altamirano A, Fernández Y, Flores R, et al. Estudio de diagnóstico nacional sobre el rezago educativo que presentan las madres jóvenes y las jóvenes embarazadas en relación con la educación básica. Informe final. México: CRIM/UNAM; 2010 [cited 2017 Nov 5]. Available from: http://www.transparenciapresupuestaria. gob.mx/work/models/PTP/programas/sed/ evaluaciones/2010/11s108c0010.pdf

23. De Jesús D, González G. Elementos teóricos para el análisis del embarazo adolescente. Sexualidad, salud y sociedad. Rev Lat [Internet]. 2014 agosto [cited 2017 Nov 2];17:98-123. Available from: http://www.scielo.br/pdf/sess/n17/1984-6487sess-17-0098.pdf 
24. Pacheco CI. Agencia social, sexualidad y embarazo en menores de 15 años. Rev Gerenc Polit Salud [Internet]. 2015 nov 1 [cited 2017 Nov 12];14(29):6282. Available from: http://www.scielo.org.co/pdf/ rgps/v14n29/v14n29a05.pdf

25. Gómez CA, Montoya LP. Factores sociales, demográficos, familiares y económicos relacionados con el embarazo en adolescentes del área urbana,
Briceño, 2012. Rev Salud Pública [Internet]. 2013 marzo 12 [cited 2017 Nov 12];16(3):394-406. Available from: https://revistas.unal.edu.co/index.php/ revsaludpublica/article/view/34986/49157

Recebido: 8 de novembro de 2017

Aprovado: 11 de abril de 2018

Publicado: 24 de agosto de 2018

A Revista Baiana de Enfermagem utiliza a Licença Creative Commons - Atribuição-NãoComercial 4.0 Internacional.

https://creativecommons.org/licenses/by-nc/4.0/

Este artigo é de acesso aberto distribuído sob os termos da Licença Creative Commons (CC BY-NC).

Esta licença permite que outros remixem, adaptem e criem a partir do seu trabalho para fins não comerciais.

Embora os novos trabalhos tenham de lhe atribuir o devido crédito e não possam ser usados para fins comerciais, os usuários não têm de licenciar esses trabalhos derivados sob os mesmos termos. 